\title{
Evaluation of Myocardial Injury Patterns and ST Changes among Critical and Non-critical Patients with Coronavirus-19 Disease
}

\author{
Anam Liaqat ( $\nabla$ dranum68@gmail.com ) \\ Riphah International University \\ Rao Saad Ali-Khan \\ Military Hospital \\ Muhammad Asad \\ Armed Forces Institute of Cardiology \\ Zakia Rafique \\ Riphah International University \\ Syed Shahzad Hasan \\ University of Huddersfield \\ Amy T Page \\ Monash University \\ Xiwen Simon Qin \\ Monash University
}

\section{Research Article}

Keywords: Coronavirus-2019, critical, echocardiogram, myocardial injury

Posted Date: November 13th, 2020

DOl: https://doi.org/10.21203/rs.3.rs-106298/v1

License: (c) (i) This work is licensed under a Creative Commons Attribution 4.0 International License.

Read Full License

Version of Record: A version of this preprint was published at Scientific Reports on March 1st, 2021. See the published version at https://doi.org/10.1038/s41598-021-84467-4. 


\section{Abstract}

Background. Novel coronavirus disease (COVID-19) has led to a major public health crisis globally. Currently, myocardial damage is speculated to be associated with COVID-19, which can be seen as one of the main causes of death of patients with COVID-19. Therefore, in this study, we aim to investigate the effects of COVID-19 diagnosed patients on myocardial injury.

Methods. A prospective study was conducted among 201 patients with COVID-19 in the Pakistan Military Hospital from April 1 to August 31, 2020, including non-critical cases and critical cases. COVID-19 patients were stratified as critical and non-critical according to signs and symptoms with those requiring intensive care and ventilator support as critical and those don't require ventilator support as non-critical

Results: A total of 201 COVID-19 patients with critical and non-critical categories presented with myocardial injury. All patients with myocardial injury had an elevation in CKMB and Trop 1 levels. Of these patients, $43.7 \%$ presented with new electrocardiography (ECG) changes, ST depression is observed in $36.3 \%$ patients, and $16.9 \%$ presented with abnormal electrocardiogram findings, with right ventricular dilatation and dysfunction. Results analyzed by a logistic regression model showing COVID-19 direct contribution to myocardial injury in these patients.

Conclusion: COVID-19 disease directly leads to cardiovascular damage among critical and non-critical patients. Myocardial injury is associated not only with abnormal ECG changes but also with myocardial dysfunction on echocardiography and more commonly observed among critical patients.

\section{Introduction}

Coronavirus disease 2019 (COVID-19) has emerged in the world as both a pandemic and a major public health crisis in China, as well as for the international community. Globally, as of there have been 49,578,590 confirmed cases of COVID-19, including 1,245,717 deaths, reported to WHO recently [1]. Serious acute respiratory syndrome coronavirus 2 (SARS-CoV-2) can be spread from person to person via respiratory droplets and direct contact, creating a major public health challenge [2]. In addition to concentrating on severe pneumonia in patients, the SARS-CoV-2 can invade several essential organs and cause multiple organ failure with cytokine storms. The heart is one of the most critical organs and it is particularly probable that SARS-COV-2 infection also contributes to viral myocardial damage, which could also be considered one of the leading causes of COVID-19 patients' death [3].

Previous studies have shown the noval insight into the occurrence and implications of SARS-COV-2 induced myocardial injury. Shi et al [4] present a retrospective study of 416 COVID-19 hospitalized patients, $19.7 \%$ of whom had evidence of myocardial injury evidenced by elevation of troponin I (Tnl) high-sensitivity levels, and higher levels of TnI were associated with higher mortality rates among those with myocardial injury. Guo et al [5] recorded related findings in 187 COVID-19 diagnosed patients, of which $27.8 \%$ had myocardial damage as calculated by high levels of troponin $\mathrm{T}$ (TnT), and the highest mortality rates were found in those with high levels of TnT that had cardiovascular disease, i.e. $69.4 \%$. 
Nevertheless, mortality rates of people with elevated levels of TnT without previous cardiovascular disease were also significant and was $37.5 \%$. Further they showed the important correlation of TnT levels with levels of C-reactive protein and N-terminal pro-B-type natriuretic peptide (NT-proBNP), thereby connecting myocardial damage to the inflammatory extent and ventricular dysfunction .Yang and Zin [6] follow up on these lines of evidence. They observed that patients having pre-existing cardiovascular conditions are more prone to adverse effects of COVID-19 disease. Notably, Shi et al, Guo et al, and Yang and Zin's papers from China also discussed the unique marked sensitivity of SARS-CoV-2 to the host angiotensin-converting enzyme 2 (ACE-2) receptor, increasing the likelihood for direct viral endothelium and myocardium infection and myocarditis.

At present, there is insufficient evidence available to resolve any specific threats of COVID-19 induced myocardial injury. This awareness is of paramount importance to understand the risk for direct and indirect adverse effects of the SARS-CoV-2 on the heart, particularly in those with already existing heart disease. In this study, we aimed to investigate the myocardial injury patterns, ECG changes, echocardiographic parameters in COVID-19 diagnosed patients of critical and non-critical categories in Pakistan's largest tertiary care center. These results will provide updated information for physicians to improve the survival of people with COVID-19.

\section{Methods}

\section{Study design and population}

This prospective study is an observational single-center which was conducted at the Military Hospital, Rawalpindi, Pakistan, one of the largest tertiary teaching hospitals, declared as the official center for COVID-19 patients. We included people who had COVID-19 hospitalizations from April 2020 till August 2020. Clinical information was obtained by the attending physician during hospitalization.

This study complies with the edicts of the 1975 Declaration of Helsinki [7], and approval was taken by the institutional ethics board of the Military Hospital of Pakistan Reference number A/28/BC/215/2020. Informed consent was taken from the attendants and stable patients.

\section{Diagnosis and grading of COVID-19}

All patients were diagnosed and graded as per the World Health Organization's interim guidance [8]. The diagnosis of COVID-19 was confirmed with real-time reverse transcriptase-polymerase chain reaction (RTPCR). A chest computed tomographic (CT) scan was performed in all positive patients to see pathological changes in the lungs. COVID-19 patients were divided into two groups as non-critical and critical patients. Critical patients were included: 1) Patients with one of the three conditions; respiratory failure (involves mechanical ventilation), shock, and organ failure (requires intensive care) [9]: 2) suffering from high respiratory frequency (RR $\geq 30 \mathrm{bpm}$ ), oxygen saturation $\leq 93 \%$, and $\mathrm{PaO} 2 / \mathrm{FiO} 2$ ratio $\leq 300 \mathrm{~mm} \mathrm{Hg}$. 
The clinical data of patients were collected from electronic medical records, including demographics, clinical symptoms, and signs, co-existing conditions, imaging findings, laboratory results.

\section{Myocardial injury patterns}

Myocardial injury was defined as a rise and fall in cardiac troponin (cTn) with at least one value above the 99th percentile upper reference limit (URL), attributable to cardiovascular and non-cardiovascular causes [10], and myocarditis related abnormalities defined as a triple elevation in cardiac Troponin I (over $0.12 \mathrm{ng} / \mathrm{mL}$ ) plus abnormalities on echocardiography and/or ECG according to American Heart Association (AHA) [11].

ECG irregularities are described as variations in ST-segment elevation / ST-T. ST-T changes were analyzed as per Minnesota Coding [12].

\section{Statistical analysis}

Categorical variables are presented as percentages, and continuous variables as mean (SD) and median. Categorical variables are gender, symptoms such as chest pain, SOB, comorbidities (Cardiovascular disease, Chronic Lung disease, smoking, obesity, Hypertension, diabetes) ECG pattern, Echocardiographic findings, lung support during hospitalization. The Chi-Squared exact test for categorical variable and independent t-test for continuous variables were used. The differences in the means for Trop-I, D-dimer, C-reactive protein CRP, creatinine phosphokinase CPK, creatinine kinase-MB CKMB, Serum Urea, age temperature, respiratory rate, body mass index BMI, number of co-morbidities were analyzed using independent t-test. Multivariate logistic regression analysis was used to assess the association between severity status (critical and non-critical) and cardiac markers (Trop-I or CKMB). All statistical analyses will be performed with SPSS, version 24.0 with 0.05 as a level of significance.

\section{Results}

\section{Demographic and clinical characteristics}

There were 201 hospitalized patients with COVID-19 disease (Table 1), including 119 male (59.2\%) and $82(40.8 \%)$ female. The mean age was $44.62 \pm 15.2$, and the mean BMI was $24.73 \pm 2.77$. There were 144 $(71.6 \%)$ patients presented as non-critical and $57(28.3 \%)$ as critical categories. Overall more males than females were affected and males constituted the majority of the critical patients' group. The most common symptoms were fever [100.3 \pm 1.1$]$, chest pain/tightness [22(38.6\%)], and shortness of breath [57(100\%)] identified among patients of the critical group as compared to patients in the non-critical group. However, respiratory rate and systolic blood pressure were higher in both critical and non-critical patients with mean and SD values of [26.5 $\pm 1.3 / 21.7 \pm 2.3]$ and [138.6 $\pm 7.8 / 125.2 \pm 9]$ respectively. P-value was $<0.001$. Critical patients were found to have more comorbidities than non-critical ones ( $p$-value <0.001). Of 201 patient, common co-existing conditions were diabetes in $35(17.5 \%)$, hypertension 35 (17.5\%) and Cardiovascular diseases 30(14.9\%) patients. 


\begin{tabular}{|c|c|c|c|c|}
\hline \multicolumn{5}{|c|}{ Table1: Demographics and Clinical characteristics of Covid-19 patients } \\
\hline CHARACTERISTICS & TOTAL & CRITICAL & NON-CRITICAL & $P$-VALUE \\
\hline No. of patients & 201 & 57 & 144 & \\
\hline Age (years) & $44.6 \pm 15.2$ & $59.4 \pm 10.9$ & $39.1 \pm 12.7$ & $<0.001$ \\
\hline Male & $119(59.2)$ & $34(61.8)$ & $85(58.2)$ & 0.643 \\
\hline Female & $82(40.8)$ & $21(38.2)$ & $61(41.8)$ & \\
\hline SOB & $77(38.3)$ & $57(100.0)$ & $20(13.9)$ & $<0.001$ \\
\hline Chest pain & $23(11.4)$ & $22(38.6)$ & $1(0.7)$ & $<0.001$ \\
\hline BMI (kg/sqm) & $24.7 \pm 2.8$ & $27.3 \pm 2.7$ & $23.8 \pm 2.1$ & $<0.001$ \\
\hline Temperature (F) & $99.0 \pm 1.1$ & $100.3 \pm 1.1$ & $98.5 \pm 0.7$ & $<0.001$ \\
\hline Respiratory rate (per min) & $23.0 \pm 2.9$ & $26.5 \pm 1.3$ & $21.7 \pm 2.3$ & $<0.001$ \\
\hline Diastolic BP (mmHg) & $79.8 \pm 7.9$ & $86.2 \pm 7.9$ & $77.5 \pm 6.4$ & $<0.001$ \\
\hline Systolic blood pressure (mmHg) & $128.9 \pm 10.5$ & $138.6 \pm 7.8$ & $125.2 \pm 9.0$ & $<0.001$ \\
\hline Blood saturation of oxygen (\%) & $96.9 \pm 2.3$ & $95.7 \pm 2.9$ & $97.4 \pm 1.8$ & $<0.001$ \\
\hline \multicolumn{5}{|l|}{ COMORBIDITIES } \\
\hline Hypertension & $35(17.5)$ & $6(3)$ & $29(14.4)$ & $<0.001$ \\
\hline Diabetes & $35(17.5)$ & $2(1)$ & $33(16.4)$ & $<0.001$ \\
\hline Cardiovascular disease & $30(14.9)$ & $3(1.5)$ & $27(13.4)$ & $<0.001$ \\
\hline Chronic lung disease & $5(2.5)$ & $0(0.0)$ & $5(2.5)$ & 0.002 \\
\hline Smoking & $33(16.4)$ & $13(6.5)$ & 20(10) & $<0.001$ \\
\hline Obesity & $10(5)$ & $0(0.0)$ & 10(10) & $<0.001$ \\
\hline Cancer & $8(4)$ & $4(2.0)$ & $4(2.0)$ & 0.242 \\
\hline
\end{tabular}

Evaluation of cardiac markers 
Trop-I were raised over $(0.04 \mathrm{ng} / \mathrm{ml})$ [Mean \pm SD $0.07 \pm 0.12$ ] among non-critical patients and triple elevated over $(0.12 \mathrm{ng} / \mathrm{ml})$ among critical group patients during hospitalization $(p<0.001)$. LDH, DDimers, Ferritin were also raised among the critical group compared to the non-severe group, showing poor prognosis in these patients. Patients in both groups showed elevated CRP with $p=0.001$. Critical patients had significantly low WBC counts and platelet counts ( $p$ value $=0.001$ ). ALT and ALP also showed an increased mean value among critical patients. Lymphocyte counts were lower in the critical group with $\mathrm{p}$ value $<0.001$ [Table 2].

Table 2: Comparison of specific and non-specific cardiac injury markers and laboratory parameters between critical and non-critical cases 


\begin{tabular}{|c|c|c|c|}
\hline Laboratory Parameters & Critical group & Non-critical group & P-value \\
\hline Troponin I (<0.04ng/ml) & $0.48 \pm 0.32$ & $0.07 \pm 0.12$ & 0.001 \\
\hline \multicolumn{4}{|l|}{ Poor prognosis>0.12 ng/ml } \\
\hline D-Dimer (<500ng/mL) & $1089.35 \pm 409.21$ & $304.87 \pm 164.55$ & 0.001 \\
\hline \multicolumn{4}{|l|}{ Poor-prognosis> 1000ng/ml } \\
\hline CRP $(<8 m g / L)$ & $153.15 \pm 72.29$ & $20.81 \pm 28.63$ & 0.001 \\
\hline \multicolumn{4}{|l|}{ Poor prognosis $>100 \mathrm{mg} / \mathrm{ml}$} \\
\hline LDH (units/L) & $528.65 \pm 211.81$ & $169.55 \pm 100.88$ & 0.001 \\
\hline Ferritin (mcg/L) & $1417.15 \pm 525.97$ & $197.14 \pm 245.45$ & 0.001 \\
\hline CPK (<192 units/L) & $213.15 \pm 17.87$ & $163.28 \pm 20.02$ & 0.001 \\
\hline CK-MB (<25U/I) & $45.56 \pm 12.17$ & $23.04 \pm 8.83$ & 0.001 \\
\hline WBC (cells/cmm) & $5.26 \pm 2.26$ & $6.58 \pm 1.38$ & 0.001 \\
\hline Absolute Lymphocyte Count (microL) & $1484.95 \pm 836.95$ & $3717.0 \pm 1163.69$ & 0.001 \\
\hline Platelets (cells/cmm) & $127.63 \pm 33.33$ & $253.22 \pm 71.36$ & 0.001 \\
\hline Serum urea $(\mathrm{mmol} / \mathrm{L})$ & $4.82 \pm 1.17$ & $5.30 \pm 0.95$ & 0.003 \\
\hline Serum-creatinine (micromol/L) & $123.55 \pm 15.34$ & $89.03 \pm 13.22$ & 0.001 \\
\hline Serum Sodium (mmol/L) & $135.20 \pm 2.14$ & $138.93 \pm 8.81$ & 0.001 \\
\hline Serum potassium (mmol/L) & $4.5 \pm 0.28$ & $4.5 \pm 2.85$ & 0.837 \\
\hline Serum Bilirubin (micromol/L) & $31.82 \pm 16.10$ & $18.18 \pm 4.23$ & 0.001 \\
\hline $\operatorname{ALT}(<42 \mathrm{IU} / \mathrm{L})$ & $98.87 \pm 45.84$ & $44.01 \pm 20.92$ & 0.001 \\
\hline Alkaline Phosphatase (<150IU/L) & $143.67 \pm 25.10$ & $111.38 \pm 27.07$ & 0.001 \\
\hline
\end{tabular}




\section{ECG findings}

New ECG findings were present only in 88 patients out of 201 patients enrolled in the study, with 55/88 $(62.5 \%)$ occurred in critical cases and 33/88 (37.5\%) in non-critical cases. In addition, critical patients were found to have more abnormal ECG findings as compared to non-critical ones ( $p$-value $<0.001$ ). Anteroseptal ST depression was seen among 20(22.7\%) critical and 12(13.6\%) non-critical categories with $p<0.001$. Anteroseptal T wave inversion was observed in $12(13.6 \%)$ critical and $9(10.2 \%)$ non-critical patients and anteroseptal ST elevation was seen in 7(7.9\%) critical and 2 non-critical (2.2\%) among 88 patients with new ECG findings. However, a new Q wave was observed in 3(3.4\%) patients of the noncritical category. Right bundle branch block (RBBB) was present in $16(18.1 \%)$ critical and $7(7.95 \%)$ noncritical group patients. Sinus tachycardia was commonly seen among COVID-19 positive patients with $43(72.8 \%)$ in critical and $16(7.9 \%)$ non-critical patients $(\mathrm{p}<0.001)$. Of 201 patients, new-onset atrial fibrillation was observed in 21(10.4), 4 patients were non-critical compared to 17 patients were critical.

\section{Echocardiography Findings}

Echo findings suggest that 25/201 patients showed RV dilatation and RV dysfunction, 5 patients with LV dysfunction only, and 4 patients reported with biventricular dysfunction of critical category. None of the non-critical group patients showed any abnormal Echo changes except in one patient with LV dysfunction. Echo findings were missing among 20/201 patients and 147/201 displayed normal echo findings. Among critical and non-critical patients $3(5.5 \%)$ VS $1(0.8 \%)$ patients showed EF less than $40 \%$, $34(61.8 \%)$ VS 18 (13.7\%) with EF between 40-59\%, and 18(32.7) VS 112(85.5\%) with EF>60\% and above respectively (Table 3 ).

Table 3: ECHO and ECG assessment by critical and non-critical cases 


\begin{tabular}{|c|c|c|c|}
\hline Items & Non-critical group & Critical group & p-value \\
\hline Normal ECG & $111(98.2)$ & $2(1.76)$ & \multirow[t]{2}{*}{0.001} \\
\hline Abnormal ECG & $33(37.5)$ & $55(62.5)$ & \\
\hline Anteroseptal ST depression & $12(13.6)$ & $20(22.7)$ & \multirow[t]{5}{*}{0.001} \\
\hline Anteroseptal ST elevation & $2(2.27)$ & $7(7.9)$ & \\
\hline Anteroseptal $T$ wave inversion & $9(10.2)$ & $12(13.6)$ & \\
\hline$Q$ wave inferior leads & $3(3.4)$ & $0(0.0)$ & \\
\hline$R B B B$ & $7(7.9)$ & $16(18.1)$ & \\
\hline Tachycardia & $16(27.1)$ & $43(72.8)$ & \multirow[t]{3}{*}{0.001} \\
\hline Atrial Fibrillation & $4(1.9)$ & $17(8.45)$ & \\
\hline First degree AV block & $1(0.4)$ & $5(2.4)$ & \\
\hline Normal ECHO & $126(99.2)$ & $21(38.9)$ & \multirow[t]{5}{*}{0.001} \\
\hline Abnormal ECHO & $1(0.8)$ & $33(61.1)$ & \\
\hline LVdysfunction & $1(0.7)$ & $4(7.0)$ & \\
\hline$R V$ dilatation, $R V$ dysfunction & $0(0.0)$ & $25(43.9)$ & \\
\hline Biventricular dysfunction & $0(0.0)$ & $4(11.7)$ & \\
\hline \multicolumn{4}{|l|}{ Ejection fraction } \\
\hline$\geq 60 \%$ & $112(85.5)$ & $18(32.7)$ & \multirow[t]{3}{*}{0.001} \\
\hline $40-59 \%$ & $18(13.7)$ & $34(61.8)$ & \\
\hline$<40 \%$ & $1(0.8)$ & $3(5.5)$ & \\
\hline Discharge patients & $144(77.0)$ & $43(22.9)$ & \multirow[t]{2}{*}{0.001} \\
\hline Death & $0(0.0)$ & $14(6.96)$ & \\
\hline
\end{tabular}

\section{Cardiac markers and risk of severity and mortality}

Table 4 presents the association between severity of COVID and cardiac enzymes/ abnormal ECHO or ECG using logistic regression. We found a significant association between severity (critical or non-critical) and cardiac markers. People with ciritical symptoms are significantly higher odds of troponin I than 
people without critical symptoms. The effect sizes were consistently large for the outcomes of CKMB, abnormal ECHO and ECG, compared to people without critical symptoms. There were 14 deaths reported in this study. All of them were critical cases with abnormal ECHO and ECG findings, and less than $60 \%$ ejection fraction.

Table 4: Adjusted odds ratios $(95 \% \mathrm{Cl})$ of cardiac injury markers, by severity status $(n=201)$

\begin{tabular}{|c|c|c|c|}
\hline \multirow[t]{2}{*}{ Items } & \multicolumn{2}{|l|}{ OR $(95 \% \mathrm{Cl})$} & \multirow[t]{2}{*}{ p-value } \\
\hline & Non-critical cases & Critical cases & \\
\hline \multirow[t]{2}{*}{ Elevated Troponin I (>0.04ng/ml) } & $\mathrm{n} / \mathrm{N}=68 / 144$ & $n / N=55 / 57$ & \\
\hline & 1.0 & $4.10(0.69-24.39)$ & 0.120 \\
\hline \multirow[t]{2}{*}{ Elevated Troponin I (>0.12ng/ml) } & $\mathrm{n} / \mathrm{N}=11 / 144$ & $\mathrm{n} / \mathrm{N}=45 / 57$ & \\
\hline & 1.0 & $8.14(2.13-31.10)$ & 0.002 \\
\hline \multirow{2}{*}{$\begin{array}{l}\text { Elevated CKMB } \\
(\geq 25 \mathrm{U} / \mathrm{I})\end{array}$} & $\mathrm{n} / \mathrm{N}=50 / 144$ & $n / N=56 / 57$ & \\
\hline & 1.0 & $10.56(1.15-96.63)$ & 0.037 \\
\hline \multirow[t]{2}{*}{ Abnormal ECHO } & $\mathrm{n} / \mathrm{N}=1 / 127$ & $\mathrm{n} / \mathrm{N}=33 / 54$ & \\
\hline & 1.0 & $5.21(0.47-57.84)$ & 0.179 \\
\hline \multirow[t]{2}{*}{ Abnormal ECG } & $n / N=33 / 144$ & $\mathrm{n} / \mathrm{N}=55 / 57$ & \\
\hline & 1.0 & $28.22(4.04-196.81)$ & 0.001 \\
\hline
\end{tabular}

ORs were adjusted for age, gender, BMI, and comorbidity. Logistic regression. Non-critical was referent

\section{Discussion}

Coronaviruses belong to the ribonucleic acid (RNA) viruses' family named Coronaviridae, present in both humans and mammals. It is an enveloped virus that can cause respiratory, enteric, hepatic, and neurologic disease. Although it has been linked to diseases involving the pulmonary tract, there is also an association with cardiac pathologies [13]. Special attention was paid to the function of angiotensinconverting enzyme 2 (ACE2), a protein that is proposed to be the binding receptor for SARS-CoV-2 and allows its cellular entrance. ACE2 is found in epithelial cells of lung alveoli, and also highly expressed in adult human heart pericytes, suggesting an inherent heart vulnerability to SARS-CoV-2 infection. Despite ACE2 mediated entry, SARS-CoV-2 also down-regulates the expression of ACE2 receptors, resulting in a diminished conversion of angiotensin II (Ang-II) to cardioprotective angiotensin 1-7 protein. Besides the heart and lungs, Ang-II conversion to 1-7 in vascular endothelium, intestinal epithelium, and the kidneys leads to the inhibition of vasoconstrictor, pro-inflammatory, pro-oxidant, pro-proliferative, and pro-fibrotic 
functions mediated by Ang-II through AT1 receptors [14]. Thus, suppression of ACE2 expression and subsequent rise in Ang-II levels in COVID-19 patients may pose a further danger to both the heart and vessels.

Although mainly research has been done on pulmonary complications, few investigations have been done to evaluate the cardiovascular effects associated with Covid-19. Elevated cardiac troponin has been observed since the first data analyses in China, representing myocardial damage as a potential pathogenic pathway that contributes to disease severity and mortality among COVID-19 patients. The phenomenon of elevated cTn levels in COVID-19 patients may be explained by several mechanisms such as viral myocarditis, myocardial damage driven by cytokine syndrome, microangiopathy, and unmasked coronary artery disease. It has been proposed that microvascular injury in patients with COVID-19 causes perfusion disorders, hyper-permeability of the vessels, and vasospasm, resulting in myocardial injury [15].

Research conducted by Wang Z et al demonstrated the association between clinical comorbidities and disease severity among COVID-19 disease patients [16]. In our study, there was a significant association between clinical comorbidities and disease severity including diabetes, hypertension, obesity, and smoking. However, cancer patients did not show any significance. Although Robilotti et al [17], showed that there was a substantial rate (20\%) of severe respiratory outcomes among cancer patients, there study results were contradictory to our findings. Evidence suggests that the development of cancer is linked with a blunted immune status depicted by an increase in immunosuppressive cytokines along with suppressed induction of pro-inflammatory danger signals, impairment in the maturation of dendritic cell, and role of immunosuppressive leukocytes [18].

Studies have shown that elevated troponin levels may be seen in 7-17\% of patients hospitalized with COVID-19 and 22-31\% of those admitted to the intensive care unit [19]. In this study, out of 201 patients, $54 \%$ had raised Trop-I levels suggesting myocardial injury, and $47.2 \%$ patients in the critical group. Trop-I were significantly higher $>0.2 \mathrm{ng} / \mathrm{ml}$ in critical patients, eventhough mean values were raised in both both groups. Increased levels of cTn were also associated with abnormal ECG findings. Other studies have also shown that heart injury and elevated troponin may contribute to complications including ventricular fibrillation, acute coagulopathy, electrolyte disturbances, acute kidney failure, and the necessity for mechanical ventilation [20]. Furthermore, the autopsy report of people who died due to the 2002 SARS outbreak revealed that $35 \%$ of heart specimens demonstrated the existence of viral RNA in the myocardium, and this, in turn, was linked to lower expression of ACE2 protein [21], SARS-CoV-2 can represent the same mechanism because the two viruses seem to be similar in the genome [22]. Also, patients with pre-existing or with risk factors for coronary artery disease have a high chance of developing severe coronary syndrome following acute infections, as shown in epidemiological and clinical influenza studies and other acute inflammatory conditions. This may arise due to an imbalance between myocardial oxygen supply and demand, such that the troponin elevation may be viewed as a type 2 myocardial infarction (MI). Decreased oxygen supply in COVID-19 patients is usually triggered by hypoxic respiratory failure and is a sign of disease severity. Infectious conditions, by comparison, are 
frequently followed by fever, tachycardia, and endocrine dysregulation, contributing to a marked rise in demand for myocardial oxygen. Besides, hypoxemia also results in elevated intracellular calcium with subsequent apoptosis of the cardiac myocytes [23].

Circulating cytokines produced during extreme systemic inflammatory stress may result in instability and breakup of atherosclerotic plaque with thrombus formation contributing to type $1 \mathrm{MI}$ due to COVID-19 [24]. Furthermore, due to the expression of ACE2 in endothelial vascular cells, a direct viral vascular infection that contributes to plaque instability also contributes to type1 MI in patients with COVID-19 [25].

Coagulopathies seen in severe COVID 19 cases can be explained by endothelial dysfunction, cytokine storm, oxidative stress, and activation of Ang II. A postmortem report from Singapore states that 4 out of 8 patients infected with SARS had pulmonary thrombo-embolic lesions and 3 had deep vein thrombosis. To date, only 1 case of pulmonary embolism associated with COVID-1939 has been reported, but only half of the COVID-19 patients have raised levels of D Dimer which is associated with more deaths [2627]. Increased D-dimer levels can be explained by inflammatory reactions, which stimulate severe fibrinolysis in the lungs with overspill into the bloodstream [28]. In our study, analysis of laboratory results revealed that D-dimers and inflammatory markers were significantly raised in critical patients. Also, critical patients presented with significantly low levels of WBC count, platelet counts, Lymphocyte counts. ALT and AST also showed increased mean values among critical patients.

Although, the incidence of the acute coronary syndrome and $\mathrm{Ml}$ in infected patients during the first SARS outbreak was described. However, in COVID-19, very limited details are available on the changes in electrocardiogram linked to MI. Angeli F et al [29] showed from their study on 50 patients that ECG changes were present in patients with COVID-19 disease irrespective of the severity of the disease. Common ECG changes were seen in $43.7 \%$ of patients in our study, Sinus tachycardia was the common followed by Anteroseptal ST Depressions irrespective of disease severity. ECG changes also when correlated with the troponin level suggested that their values were higher as compared to ones having normal ECG ( $p$-value $<0.001$ ). Also, ECG changes had a significant relation with the mortality as well ( $p$ value $<0.001)$ among COVID-19 disease patients which are positively related to the severity of the disease. Furthermore, among patients with abnormal ECG patterns, we observed RV dilatation and RV dysfunction commonly among critical group category patients suggesting SARS-COV infection severity in cardiac damage, especially among critical group patients.

\section{Limitations}

There are a few limitations to our study. This was a single-center experience with a relatively small sample size therefore, further studies with a large population size may help to draw a definitive conclusion regarding the severity of disease and ECG changes. Further, a detailed evaluation of echocardiographic changes was not studied owing to the short study period and the limited amount of time due to the disease's peak period going on, so observing more parameters with better modalities might give a broader picture of cardiac involvement. In addition, the reversibility of the electrocardiographic changes with an improvement of the clinical status could not be discerned. 


\section{Conclusions}

COVID-19 disease is associated with multi-system involvement including the cardiovascular system. Ecg changes are commonly observed in patients belonging to the severe category. Myocardial injury is also associated, not only with abnormal ECG changes but also with myocardial dysfunction on echocardiography. Death was also common among patients showing these injury patterns. Cardiac Involvement can be considered to change the course of the disease so close assessment of cardiac changes should be taken into account especially in critical patients.

\section{Declarations}

CONTRIBUTORS: Project conceptualization and preparation/drafting of the manuscript: Anam Liaqat, Rao Saad Ali-Khan and Muhammad Asad; statistical analysis: Zakia Rafique, Hasan Shahzad, Amy Page and Xiwen Simon Qin ; data interpretation, revision/editing and proofreading of the manuscript: all authors.

\section{CONFLICT OF INTEREST}

None

\section{FUNDING}

None

\section{References}

1. Coronavirus Official Data | WHO Data on COVID-19 | who.int

2. Li, Q. et al. Early transmission dynamics in Wuhan, China, of novel coronavirus-infected pneumonia. N Engl J Med 382,1199-120 (2020).

3. Channappanavar, R. \& Perlman, S. Pathogenic human coronavirus infections: causes and consequences of cytokine storm and immunopathology. Immunopathol. 39,529-539(2017).

4. Shi, S. et al. Association of cardiac injury with mortality in hospitalized patients with COVID-19 in Wuhan, China. JAMA Cardiol. 5,802-810(2020).

5. Guo, T. et al. Cardiovascular implications of fatal outcomes of patients with coronavirus disease 2019 (COVID-19). JAMA Cardiol. 5,811-818(2020).

6. Yang, C. \& Jin, Z. An acute respiratory infection runs into the most common non-communicable epidemic-COVID-19 and cardiovascular diseases. JAMA Cardiol. 5,743-744 (2020).

7. World Medical Association. Declaration of Helsinki: ethical principles for medical research involving human subjects. JAMA. 310,2191-2194(2013).

8. Clinical Management of Severe Acute Respiratory Infection When Novel Coronavirus (nCoV) Infection is Suspected: Interim Guidance. World Health Organization. 
9. Huang, C., Wang, Y. \& Li, X. Clinical features of patients infected with 2019 novel coronavirus in Wuhan, China. Lancet. 395,497-506(2020).

10. Thygesen, K. et al. ESC Scientific document group. Fourth universal definition of myocardial infarction. Eur Heart J. 40,237-269(2019).

11. Kociol, R.D. et al. American Heart Association Heart, CT Transplantation Committee of the Council on Clinical, recognition and initial management of fulminant myocarditis: a scientific statement from the American Heart Association. Cir. 141,69-92(2020).

12. Prineas, R., Crow, R. \& Blackburn, H. The minnesota code manual of electrocardiographic findings: standards and procedures for measurement and classification. Littleton: John Wright-PSG.; 1982.

13. Driggin, E. et al. Cardiovascular considerations for patients, health care workers, and health systems during the coronavirus disease 2019 (COVID-19) pandemic. J Am Coll Cardiol. 75,2352-2371(2020).

14. Kuster, G.M. et al. SARS-CoV2: should inhibitors of the renin-angiotensin system be withdrawn in patients with COVID-19? Eur Heart J. 41,1801-1803(2020).

15. Chen, Y. et al. Potential pathophysiological mechanisms underlying COVID-19-induced myocardial injury Chin. J Pathophysiol. 36,573-576(2020).

16. Zhongliang, W. et al. Clinical Features of 69 Cases With Coronavirus Disease 2019 in Wuhan, China. Clin. Infect. Dis. 71,769-777(2020).

17. Robilotti, E.V. et al . Determinants of COVID-19 disease severity in patients with cancer. Nat Med. 26,1218-1223(2020).

18. Schreiber, R.D., Old, L.J. \& Smyth, M.J. Cancer immunoediting: integrating immunity's roles in cancer suppression and promotion. Science. 33,1565-1570(2011).

19. Huang, C., Wang, Y. \& Li, X. Clinical features of patients infected with 2019 novel coronavirus in Wuhan, China. Lancet. 395,497-506(2020).

20. Guo, T. et al. Cardiovascular implications of fatal outcomes of patients with coronavirus disease 2019 (COVID-19). JAMA Cardiol. 5,811-818 (2020).

21. Oudit, G.Y. et al (2009). SARS-coronavirus modulation of myocardial ACE2 expression and inflammation in patients with SARS. Eur J Clin Invest. 39, 618-625(2009).

22. Zhu, N. et al. A novel coronavirus from patients with pneumonia in China, 2019. N Engl J Med. 382,727-733(2020).

23. Kwong, J.C. et al. Acute myocardial infarction after laboratory-confirmed influenza infection. $N$ Engl J Med. 378,345-353(2018).

24. Zheng, Y.Y. et al. COVID-19 and the cardiovascular system. Nat Rev Cardiol. 17,259-260 (2020).

25. Hamming, I. et al. Tissue distribution of ACE2 protein, the functional receptor for SARS coronavirus. A first step in understanding SARS pathogenesis. J Pathol. 203,631-637(2004).

26. Chong, P.Y. et al. Analysis of deaths during the severe acute respiratory syndrome (SARS) epidemic in Singapore: challenges in determining a SARS diagnosis. Arch Pathol Lab Med. 128,195-204(2004). 
27. Danzi, G.B., Loffi, M. \& Galeazzi G. Acute pulmonary embolism and COVID-19 pneumonia: a random association? Eur Heart J. 41,1858(2020).

28. Tang, N., Li, D., Wang, X. \& Sun, Z. Abnormal coagulation parameters are associated with poor prognosis in patients with novel coronavirus pneumonia. J Thromb Haemos. 18,844-847(2020).

29. Angeli, F. et al. Electrocardiographic features of patients with COVID-19 pneumonia. Eur. J. Intern. Med. 78,101-106(2020). 